\title{
Update knowledge of dry mouth- A guideline for dentists
}

\author{
Alsakran Altamimi Mohammed
}

Department of restorative dental sciences, Al-Farabi College of dentistry and nursing, Riyadh, Saudi Arabia.

\begin{abstract}
Background: Dry mouth is usually caused by a reduced salivary flow or by changes in the biochemical composition of saliva.

Objective: The aim of this paper is a review of the update literature of dry mouth.

Methods: We search in pubmed in the past 10 years using the words «dry mouth», «causes», «symptoms», «treatment» and «dentistry». A large number of papers have been identified. Papers not relevant to the issue were removed reducing the entries to 56 only.

Results: There are no clearly established protocols for the treatment of dry mouth in the literature. Most of identified papers were systematic reviews, non-systematic reviews, and observational studies. The most studied patients were Sjögren's syndrome and the irradiated patients. Treatments are focused on the etiology, prevention, symptomatic, local salivary stimulation and systemic treatments.

Conclusion: It can be concluded that there is no clear evidence for the causes and treatment of dry mouth, therefore the majority of the general dental practitioners refer most of the cases to appropriate specialist. Treatment must be individualized, salivary substitutes and mechanical stimulation techniques can be applied.
\end{abstract}

Keywords: Dry mouth, xerostomia

DOI: http://dx.doi.org/10.4314/ahs.v14i3.33

\section{Introduction}

Everyone has a dry mouth once in a while, especially if you're nervous, upset or under stress. But if you have a dry mouth all or most of the time, it can be uncomfortable and can lead to more serious health problems or indicate that a more serious medical condition may exist. That's because saliva does more than just keep the mouth wetit helps digest food, protects teeth from decay, prevents infection by controlling bacteria in the mouth, and makes it possible for you to chew and swallow (1-2). Dry mouth is usually caused by a reduced salivary flow or by changes in the biochemical composition of saliva. Patients suffering from dry mouth usually complain about difficulties when chewing, swallowing or even speaking.

Drugs most commonly associated with xerostomia are: -antidepressants (particularly tricyclic antidepressants)

\section{Corresponding author: \\ Department of restorative dental sciences, Al-Farabi College of dentistry and nursing, Riyadh, Saudi Arabia \\ Mohammed Alsakran Altamimi maatamimi@yahoo.com}

(11); - Selective Serotonin Reuptake Inhibitors (SSRIs), particularly when combined with benzodiazepines (12); - Diuretics, antihypertensive drugs and angiotensinconverting enzyme inhibitors (ACE inhibitors), - oral hypoglycemiants, - acetylsalicylic acid (ASA), -iron supplements. Let us not forget that drugs with the most

intense xerostomizing effect are also the most widely and frequently used (treatment of metal disorders and cardiovascular diseases) (13).

Many medicines can cause dry mouth, including drugs used to treat obesity (14), epilepsy (15), hypertension (diuretics) (16), diarrhea (17), urinary incontinence (18), asthma (certain bronchodilators 19), and Parkinson's disease (20) table 1.

Aging older people are more likely to be taking medications that may cause dry mouth. Also, older people are more likely to have other health conditions that may cause dry mouth table 1 .

Bakke et al, (20) have reported that xerostomia was higher in women than in men in all age groups. There was higher prevalence of xerostomia with increasing age in both sexes and it was more frequent at night than during 
daytime. They concluded that the dramatic increase of xerostomia between age 50 and 75 , especially amongst women, needs to be considered in the management of this age group.

Cancer therapy Chemotherapy drugs can change the nature of saliva and the amount produced. Radiation treatments to head and neck can damage salivary glands, causing a marked decrease in saliva production (21) table 1.

Side effect of certain diseases and infections. Dry mouth can be a side effect of medical conditions, including Sjögren's syndrome, HIV/AIDS, Alzheimer's disease, diabetes, anemia, rheumatoid arthritis, hypertension table 1 .

Sjögren's syndrome is an autoimmune disease characterized by dryness of the mouth and eyes. Autoimmune diseases feature the abnormal production of extra antibodies in the blood that are directed against various tissues of the body (22). The misdirected immune system in autoimmunity tends to lead to inflammation of tissues. This particular autoimmune illness features inflammation in glands of the body that are responsible for producing tears and saliva. Inflammation of the glands that produce tears (lacrimal glands) leads to decreased water production for tears and decreased saliva production and dry eyes. Inflammation of the glands that produce the saliva in the mouth (salivary glands, including the parotid glands) leads to dry mouth and dry lips (22-23). Sjogren's syndrome with gland inflammation (resulting in dry eyes and mouth, etc.) that is not associated with another connective tissue disease is referred to as primary Sjögren's syndrome. Sjögren's syndrome that is also associated with a connective tissue disease, such as rheumatoid arthritis, systemic lupus erythematosus, or scleroderma, is referred to as secondary Sjögren's syndrome. Dryness of eyes and mouth, in patients with or without Sjögren's syndrome, is sometimes referred to as sicca syndrome (22-23).

\section{HIV infection}

The acute (primary) stage of HIV infection may be accompanied by mild, flu-like symptoms, while the longest stage of infection, latency, is asymptomatic. Oral HIV symptoms generally do not occur until stage 3, when the immune system has begun to falter but AIDS has not yet developed (24). Monitoring oral health can be a good way to trace the general development of HIV disease. A number of conditions can cause sores in or around the mouth. These include oral manifestations of cancers like Kaposi's sarcoma and lymphoma, herpes viruses, human papilloma virus, dry mouth and ulceration (25). Jeganathan et al, (25) investigated the impact of xerostomia on oral health and quality of life among patients infected with human immunodeficiency virus (HIV) who were attending for routine HIV monitoring in Australia.

They have concluded that the chronic inflammation of salivary glands despite the beneficial effects of antiretroviral therapy may play a role in the etiology of xerostomia in patients infected with HIV.

\section{Alzheimer's disease}

Alzheimer's disease is a progressive neurologic disease of the brain leading to the irreversible loss of neurons and the loss of intellectual abilities, including memory and reasoning, which become severe enough to impede social or occupational functioning (26). Alzheimer's disease can cause frequent dehydration, therefore dry mouth is a problem in Alzheimer's patients, particularly in the later stages (26).

\section{Diabetes}

Dry mouth may be a warning sign of diabetes, but is not the only symptom that indicates this condition. It is, however, common in those suffering from type 1,2 diabetes (27-28). Dry mouth may be caused as a side effect of medication that is being consumed to keep the diabetes in control. Also, several patients take medicines to keep a control on their blood pressure and to prevent heart disease that may be triggered by diabetes. These are also known to cause dry mouth in patients.

Diabetics are prone to dehydration, and one of the effects of this is dryness of the mouth (28).

Kidney Problems: Even kidney problems may be a result of diabetes, thereby throwing the level of hydration in the body off balance. Dry mouth can then be an effect of this condition (28).

Persistently High Blood Sugar Levels: High blood sugar levels or hyperglycemia also tend to affect the body in many ways, one of them being dry mouth (28).

\section{Anemia}

It is a condition that develops when your blood lacks enough healthy red blood cells or hemoglobin. Hemoglobin is a main part of red blood cells and binds oxygen. If you have too few or abnormal red blood 
cells, or your hemoglobin is abnormal or low, the cells in your body will not get enough oxygen (29).

\section{Rheumatoid Arthritis}

Rheumatoid Arthritis is a chronic, systemic disease. RA is a member of a family autoimmune diseases with a high level of rheumatoid factor which gives the condition its name. Rheumatoid Arthritis is characterized by a pattern of swollen tender joints. The joints most often include the small joints of the hands and feet, wrists and knees. Rheumatoid Arthritis often affects salivary glands causing inflamation and dryness. Sjorgren's syndrome the combination of dry eye (keratoconjunctivitis sicca) and dry mouth (xerostomia) is common in Rheumatoid Arthritis (30).

\section{Hypertension}

is generally a symptom less condition in which abnormally high pressure in the arteries increases the risk of problems such as stroke, aneurysm, heart failure, heart attack, and kidney damage (31). Habbab et al, (31) reported that the frequency of potential oral manifestations in patients receiving cardiovascular agents was $14.1 \%$. The oral symptoms and/or signs were recorded in 75 (14.1\%) patients with xerostomia being the most common $(7.5 \%)$, followed by lichenoid (lichen planus-like) lesions (3.6\%) and dysgeusia (1.9\%).

\section{Nerve damage}

injury to the head or neck can damage the nerves that tell salivary glands to make saliva (33) table 1.

\section{Dehydration}

Conditions that lead to dehydration, such as fever, excessive sweating, vomiting, diarrhea, blood loss, and burns can cause dry mouth (34) table 1.

Table 1: Shows the etiological factors of dry mouth

\begin{tabular}{|l|}
\hline Side effect of certain medications \\
\hline Aging \\
\hline Cancer therapy \\
\hline $\begin{array}{l}\text { Side effect of certain diseases and infections (Sjögren's syndrome- HIV infection- Alzheimer's disease } \\
\text { - Diabetes - anemia- Rheumatoid Arthritis- Hypertension ) }\end{array}$ \\
\hline Nerve damage \\
\hline Dehydration \\
\hline Surgical removal of the salivary glands \\
\hline Lifestyle \\
\hline
\end{tabular}

\section{Surgical removal of the salivary glands}

Cunning et al, (35) reported that the Unilateral submandibular gland excision results in a decreased rate of resting salivary flow table 1 .

\section{Lifestyle}

Smoking or chewing tobacco can affect saliva production and aggravate dry mouth. Continuously breathing with your mouth open can also contribute to the problem (36) table 1.

\section{Symptoms of Dry Mouth}

If dry mouth is not properly treated, it can lead to more serious dry mouth symptoms such as gum disease and cavities. This is because the saliva that normally protects the gums and teeth is significantly reduced or is no longer present. Lack of saliva can leave the mucosa (mucus membrane) of the mouth vulnerable to infections or decrease protection against other infections of the mouth such as thrush (yeast infection) (37-38).

Hyposalivation makes reference to a decreased salivary flow and it is, therefore, an objective and measurable variable $(39,40,41)$. Hyposalivation is considered to appear when salivary flow rates are under 0.1-0.2 $\mathrm{mL} / \mathrm{min}$ (unstimulated) or $0.7 \mathrm{~mL} / \mathrm{min}$ (stimulated). Xerostomia is often associated with hyposalivation, but not always. It is widely accepted that a salivary flow rate reduction of around $50 \%$ implies the manifestation of signs and symptoms of xerostomia $(42,43)$. However, some cases have been described in patients with a normal salivary flow rate. Furthermore, other patients with a decreased salivary flow rates (generally fewer than $50 \%$ if compared to normal levels) have not complaint about oral problems. There are many symptoms of dry mouth, some of which cause great discomfort or even pain to the sufferer. The most common dry mouth symptoms were reported thirst, saliva that seems thick, stringy, dry, sticky feeling in the mouth, Halitosis (bad breath), dry hoarseness of the throat, dry, irritable and 
scratchy tongue, burning or tingling sensation of your tongue, difficulty speaking, inability to chew, swallow or taste food, dry nasal passages, painful sores of the mouth and tongue, chapped lips, increased plaque, tooth decay and gum disease (40-43) table 2.

Table 2: shows the most common symptoms of dry mouth

\begin{tabular}{l}
\hline gum disease and cavities \\
thirst, saliva that seems thick, stringy, dry, sticky feeling in the mouth \\
\hline Halitosis (bad breath), dry hoarseness of the throat, dry \\
\hline difficulty speaking, inability to chew, swallow or taste food, dry nasal passages, painful sores of the mouth and \\
tongue, chapped lips, increased plaque
\end{tabular}

\section{Management of dry mouth}

Dry mouth might be occurred by hypertensive drugs, they can be treated with salivary stimulants (sialogogues). Treatment with systemic sialogogues with anticholinesterasic and cholinergic action represents an efficient therapeutic option, although they are usually dropped out because of the quantitative and qualitative importance of their side effects. The application of topical sialogogues can be a useful alternative in the treatment of reversible xerostomia induced by drugs, as in spite of the fact that their therapeutic effects are more transitory; their side effects are also less powerful (44). Gambon et al, (45) reported that the application of citric acid might be increased risk of caries, as a consequence of the erosive action of these agents over the dentin.

Anneroth et al, (46) reported that the use of chewing gums can cause similar bad effects. In order to avoid demineralising effect on the human dentin, the use of spray format allows a direct contact with the oral mucosa, and combined with a suitable concentration (as the stimulant effect on saliva production), could reduce the demineralising potential of chewable products (47, 48).

da Mata et al, (49) indicated that Gustatory stimulants of salivary secretion with fluoride, xylitol and lower acid content maintain similar salivary stimulation capacity while reducing significantly the dental erosion predictive potential.

\section{Discussion}

The dental practionnaires have difficulty in diagnosis and treatment of dry mouth, therefore they refer all the patients to specialist in oral medicine. Diagnosis of xerostomia may be based on evidence obtained from the patient's history, an examination of the oral cavity and/or sialometry, a procedure that measures the flow rate of saliva. Xerostomia should be considered if the patient complains of dry mouth, particularly at night, or of difficulty eating dry foods such as crackers $(50,51)$. When the mouth is examined, a tongue depressor may stick to the buccal mucosa (52). In women, the "lipstick sign," where lipstick adheres to the front teeth, may be a useful indicator of xerostomia (50-52).

The oral mucosa may be dry and sticky, or it may appear erythematous due to an overgrowth of Candida albicans. The red patches often affect the hard or soft palate and dorsal surface of the tongue. Occasionally, pseudomembranous candidiasis will be present, appearing as removable white plaques on any mucosal surface. There may be little or no pooled saliva in the floor of the mouth, and the tongue may appear dry with decreased numbers of papillae. The saliva may appear stringy, ropy or foamy. Dental caries may be found at the cervical margin or neck of the teeth, the incisal margins or the tips of the teeth (50).

Several office tests and techniques can be utilized to ascertain the function of salivary glands. In sialometry, or salivary flow measurement, collection devices are placed over the parotid gland or the submandibular. Sublingual gland duct orifices, and saliva is stimulated with citric acid. The normal salivary flow rate for unstimulated saliva from the parotid gland is 0.4 to $1.5 \mathrm{~mL} / \mathrm{min} /$ gland (50-53). The normal flow rate for unstimulated, resting whole saliva is 0.3 to $0.5 \mathrm{~mL} / \mathrm{min}$; for stimulated saliva, 1 to $2 \mathrm{~mL} / \mathrm{min}$. Values less than $0.1 \mathrm{~mL} / \mathrm{min}$ are typically considered xerostomic, although reduced flow may not always be associated with complaints of dryness (49-53). Sialography is an imaging technique that may be 
useful in identifying salivary gland stones and masses. It involves the injection of radio-opaque media into the salivary glands 950-51). Salivary scintigraphy can be useful in assessing salivary gland function. Technetium$99 \mathrm{~m}$ sodium pertechnate is intravenously injected to ascertain the rate and density of uptake and the time of excretion in the mouth (50-51). Patients complaining of xerostomia should be interviewed and their medications should be reviewed. It may be possible to change medications or dosages to provide increased salivary flow. Symptoms of xerostomia are often worse between meals, at night and in the morning. Therefore, consider modifying drug schedules to achieve maximum plasma levels when the patient is awake (52).

Management of patient with dry mouth is directed toward palliation with taken in consideration of local and systemic factors and analysis of all prescription, medications, and diet. The management of dry mouth include the use of topical agents such as oral polymer based sprays, so called saliva substitutes, sipping of small amounts of water during the day, elimination of caffeine containing products, chewing of sugar free gum, and elimination of alcohol containing mouthrinses (54-55). Oral hygiene is recommended to prevent dental caries.

Taste or gestation relates to the perception of four elements and their relationship to each other. These four elements include the modalities of sweet, salty, sour, and bitter. Alterations of taste can be minimal and only slightly bothersome to incapacitating, with possible resultant depression and anorexia as secondary clinical events (55).

The dental practitioners should perform qualitative testing of the basic taste modalities, a thorough review of a patient's medical and surgical history and careful oral examination may provide clues for possible cause of dry mouth. Oral mucosal diseases including candidiasis must be considered as important etiologic factors. Upper airway surgery, viral infection, or neoplasia may alter olfactory function, thus secondarily affecting taste perception. Tonsillectomy and orofacial orthognathic surgical procedures may produce chorda tympani injury, thus affecting at least one sided taste function. Many diseases are capable of inducing taste aberrations as are representatives of several drug classes. Patients with xerostomia, salivary stimulation with sialogogues may be useful. Patients must be counseled concerning their role in management. This includes several aspects of food intake such as increasing texture, maximizing smell, and avoiding food spoilage (56).

\section{Conclusion}

It can be concluded that there is no clear evidence for the causes and treatment of dry mouth, therefore the majority of the general dental practionnaires refer most of the cases to appropriate specialist. Treatment must be individualized, salivary substitutes and mechanical stimulation techniques can be applied.

\section{References}

1. Navazesh M, Christensen C, Brightman V. Clinical criteria for the diagnosis of salivary gland bypofunction. J Dent Res 1992; 71: 1363-9.

2. Mittal S, Bansal V, Garg S, Atreja G, Bansal S. The diagnostic role of Saliva - A Review. J Clin Exp Dent 2011; 3: e314-20.

3. Cassolato SF, Turnbull RS. Xerostomia: Clinical aspects and treatment. Gerodontology 2003; 20: 64-77.

4. Gupta A, Epstein JB, Sroussi H. Hyposalivation in elderly patients. J Can Dent Assoc 2006; 72: 841-6.

5. Silvestre-Donat FJ, Miralles-Jorda L, Martinez-Mihi V. Protocol for the clinical management of dry mouth. Med Oral 2004; 9:273-9.

6. Scully C, Bagan JV. Adverse drug reactions in the orofacial region. Crit Rev Oral Biol Med 2004; 15:221-39.

7. Martín-Piedra MA, Gómez-Moreno G, Herrera $\mathrm{D}$, Aguilar-Salvatierra A. Effectiveness of a recent topical sialogogue in the management of drug-induced xerostomia. J Clin Exp Dent 2011; 3:e268-73.

8. Howland RH. Potential adverse effects of discontinuing psychotropic drugs. Part 1: Adrenergic, cholinergic, and histamine drugs. J Psychosoc Nurs Ment Health Serv 2010 ;48:11-4.

9. Jovanović S, Gajić I, Mandić B, Mandić J, Radivojević V. Oral lesions in patients with psychiatric disorders. Srp Arb Celok Lek. 2010; 138: 564-9.

10. Bergdahl M, Bergdahl J. Low unstimulated salivary flow and subjective oral dryness: association with medication, anxiety, depression, and stress. J Dent Res 2000; 79: 1652-8.

11. Murray Thomson W, Chalmers JM, John Spencer A, Slade GD, Carter KD. A longitudinal study of medication exposure and xerostomia among older people. Gerodontology. 2006; 23: 205-13.

12. De Almeida Pdel V, Grégio AM, Brancher JA, et al. Effects of antidepressants and benzodiazepines on stimulated salivary flow rate and biochemistry composition of the saliva. Oral Surg Oral Med Oral Pathol Oral Radiol Endod. 2008; 106: 58-65.

13. Thomson WM, Chalmers JM, Spencer AJ, Williams 
SM. The Xerostomia Inventory: a multi-item approach to measuring dry mouth. Community Dent Health 1999; 16: 12-7.

14. Locker D. Subjective reports of oral dryness in an older adult population. Community Dent Oral Epidemiol. 1993; 21: 165-8.

15. Bondarenko II, Kissin MIa. The use of toreal in the monotherapy of epilepsy in adults. Zh Nevrol Psikhiatr Im S S Korsakova 2009; 109: 35-9.

16. Nonzee V, Manopatanakul S, Khovidhunkit SO. Xerostomia, byposalivation and oral microbiota in patients using antibypertensive medications. I Med Assoc Thai 2012; 95:96104.

17. Astrup A, Madsbad S, Breum L, Jensen TJ, Kroustrup JP, Larsen TM. Effect of tesofensine on bodyweight loss, body composition, and quality of life in obese patients: a randomised, double-blind, placebo-controlled trial. Lancet 2008; 29: $1906-$ 13. doi: 10.1016/S0140-6736(08)61525-1.

18. Rai BP, Cody JD, Alhasso A, Stewart L. Anticholinergic drugs versus non-drug active therapies for non-neurogenic overactive bladder syndrome in adults. Cochrane Database Syst Rev 2012; 12: CD003193. doi: 10.1002/14651858.CD003193.

19. Godara N, Godara R, Khullar M. Impact of inhalation therapy on oral health. Lung India 2011; 28: 272-5. doi: 10.4103/0970-2113.85689.

20. Bakke M, Larsen SL, Lautrup C, Karlsborg M. Orofacial function and oral bealth in patients with Parkinson's disease. Eur J Oral Sci 2011; 119: 27-32. doi: 10.1111/ j.1600-0722.2010.00802.x.

21. Wang R, Wu F, Lu H, et al. Definitive intensity-modulated radiation therapy for nasopharyngeal carcinoma: long-term outcome of a multicenter prospective study. I Cancer Res Clin Oncol 2012; 18.

22. Feltsan T, Stanko P, Mracna J. Sjögren's syndrome in present. Bratisl Lek Listy 2012; 113: 514-6.

23. Castro I, Sepúlveda D, Cortés J, et al. Oral dryness in Sjögren's syndrome patients. Not just a question of water. Autoimmun Rev 2012; 30: 567-74.

24. Khan SA, Moorthy J, Omar H, Hasan SS. People living with HIV I AIDS (PLWHA) and HIV / AIDS associated oral lesions; A study in Malaysia. BMC Public Health; 2012; 12: 850 .

25. Jeganathan S, Carey H, Purnomo J. Impact of xerostomia on oral bealth and quality of life among adults infected with HIV-1. Spec Care Dentist. 2012; 32: 130-5.

26. Brayne C, Davis D. Making Alqheimer's and dementia research fit for populations. Lancet 2012; 20;380(9851):1441-3.

27. Yeh CK, Harris SE, Mohan S, Horn D, Fajardo $\mathrm{R}$, Chun YH, et al. Hyperglycemia and xerostomia are key determinants of tooth decay in type 1 diabetic mice. Lab Invest 2012; 92: 868-82.
28. BajajS, Prasad S, Gupta A, Singh VB. Oralmanifestations in type-2 diabetes and related complications. Indian I Endocrinol Metab 2012; 16: 777-9.

29. Shimoji S, Takiuchi Y, Maruoka H, Inoue D, Kimura T, Mori M, et al. Exacerbation of autoimmune neutropenia to agranulocytosis in association with severe autoimmune thrombocytopenia and hemolytic anemia in a patient with Sjögren's syndrome. Rinsho Ketsueki 2011; 52: 535-9.

30. Oğütcen-Toller M, Gedik R, Gedik S, Göze F. Sjögren's syndrome: A case report and review of the literature. West Indian Med J. 2012 ; 61:305-8.

31. Riley CK, Terezhalmy GT. The patient with hypertension. Quintessence Int 2001; 32 : 671-90.

32. Habbab KM, Moles DR, Porter SR. Potential oral manifestations of cardiovascular drugs. Oral Dis $2010 ; 16$ : 769-73.

33. Vissink A, Mitchell JB, Baum BJ, Limesand KH, Jensen SB, Fox PC, et al. Clinical management of salivary gland bypofunction and xerostomia in head-and-neck cancer patients: successes and barriers. Int J Radiat Oncol Biol Phys. 2010 15;78(4):983-91.

34. Visvanathan V, Nix P. Managing the patient presenting with xerostomia: a review. Int J Clin Pract 2010; 64: 404-7. doi: 10.1111/j.1742-1241.2009.02132.x.

35. Cunning DM, Lipke N, Wax MK. Significance of unilateral submandibular gland excision on salivary flow in noncancer patients. Laryngoscope 1998; 108: 812-5.

36. Singh M, Tonk RS. Xerostomia: Etiology, diagnosis, and management. Dent Today 2012; 31: 80, 82-3; quiz 84-5.

37. Vissink A, Bootsma H, Kroese FG, Kallenberg CG. How to assess treatment efficacy in Sjögren's syndrome? Curr Opin Rheumatol 2012; 24: 281-9.

38. Glore RJ, Spiteri-Staines K, Paleri V. A patient with dry mouth. Clin Otolaryngol. 2009; 34: 358-63.

39. Cassolato SF, Turnbull RS. Xerostomia: Clinical aspects and treatment. Gerodontology 2003; 20:64-77.

40. Navazesh M, Christensen C, Brightman V. Clinical criteria for the diagnosis of salivaly gland bypofunction. J Dent Res 1992; 71:1363-9.

41. Mittal S, Bansal V, Garg S, Atreja G, Bansal S. The diagnostic role of Saliva -A Review. J Clin Exp Dent 2011; 3:e314-20.

42. Dawes C. Physiological factors affecting salivary flow rate, oral sugar clearance, and the sensation of dry mouth in man. J Dent Res 1987; 66: 648-53.

43. Wolff MS, Kleinberg I. The effect of ammonium glycopyrrolate (Robinul)-induced xerostomia on oral mucosal wetness and flow of gingival crevicular fluid in bumans. Arch Oral Biol 1999; 44:97- 102.

44. Navazesh M, Christensen CM. A comparison of whole mouth resting and stimulated salivary measurement procedures. J 
Dent Res 1982; 61:1158-62.

45. Gambon DL, Brand HS, Nieuw Amerongen AV. The erosive potential of candy sprays. Br Dent J 2009; 206:530-1. 46. Anneroth G, Nordenram G, Bengtsson S. Effect of saliva stimulants (Hybrin and malic acid) on cervical root surfaces in vitro. Scand J Dent Res 1980; 88:214-8.

47. Chunmuang S, Jitpukdeebodintra S, Chuenarrom C, Benjakul P. Effect of xylitol and fluoride on enamel erosion in vitro. J Oral Sci 2007; 49: 293-7.

48. Hove LH, Holme B, Young A, Tveit AB. The protective effect of TiF4, SnF2 and NaF against erosion-like lesions in situ. Caries Res 2008; 42: 68-72.

49. Da Mata AD, da Silva Marques DN, Silveira JM, Marques JR, de Melo Campos Felino ET, Guilherme NF. Effects of gustatory stimulants of salivary secretion on salivary $\mathrm{pH}$ and flow: a randomized controlled trial. Oral Dis
2009; 15: 220-8.

50. Greenspan D. Xerostomia: Diagnosis and management. Oncology 1996; 10: 7-11.

51. Sreebny LM, Valdini A. Xerostomia: A neglected symptom. Arch Intern Med 1987; 147:1333-1337.

52. McDonald E, Marino C. Dry mouth: Diagnosing and treating its multiple causes. Geriatrics 1991; 46: 61-63.

53. Dyke S. Clinical management and review of Sjögren's syndrome. Int J Pharm Compound 2000; 4: 338-341.

54. Cassolato SF, Turnbull SF. Xerostomia: Clinical aspects and treatment, gerodontology 2003; 20: 64-77.

55. Porter SJ, Scully C, hegarty AM. An update on the etiology and management of xerostomia. Oral surg Oral med Oral pathol oral radiol Endod 2004; 97: 28-46.

56. Guggenheimer J, Moore P. Xerostomia. Etiology, recognition and treatment. JADA 2003; 134: 61-69. 